TRANSACTIONS OF THE

AMERICAN MATHEMATICAL SOCIETY

Volume 349, Number 9, September 1997, Pages 3585-3596

S 0002-9947(97)01852-7

\title{
ABSOLUTE BOREL SETS AND FUNCTION SPACES
}

\author{
WITOLD MARCISZEWSKI AND JAN PELANT
}

\begin{abstract}
An internal characterization of metric spaces which are absolute Borel sets of multiplicative classes is given. This characterization uses complete sequences of covers, a notion introduced by Frolík for characterizing Cechcomplete spaces.

We also show that the absolute Borel class of $X$ is determined by the uniform structure of the space of continuous functions $C_{p}(X)$; however the case of absolute $G_{\delta}$ metric spaces is still open. More precisely, we prove that, for metrizable spaces $X$ and $Y$, if $\Phi: C_{p}(X) \rightarrow C_{p}(Y)$ is a uniformly continuous surjection and $X$ is an absolute Borel set of multiplicative (resp., additive) class $\alpha, \alpha>1$, then $Y$ is also an absolute Borel set of the same class. This result is new even if $\Phi$ is a linear homeomorphism, and extends a result of Baars, de Groot, and Pelant which shows that the Čech-completeness of a metric space $X$ is determined by the linear structure of $C_{p}(X)$.
\end{abstract}

\section{INTRODUCTION}

Let us recall that a metrizable space $X$ is an absolute Borel set of additive class $\alpha, \alpha>1$ (resp., multiplicative class $\alpha, \alpha>0$ ) if $X$ embedded into an arbitrary metric space $M$ is a Borel subset of $M$ of that given class, see [Ku]. In particular, absolute Borel sets of multiplicative class 1 , i.e. absolute $G_{\delta}$-sets, are completely metrizable spaces.

The notion of complete sequences of covers was used by Z. Frolík to give internal characterizations of Čech-complete (hence topological absolute $G_{\delta}$ ) spaces and $K$ analytic spaces (see [F1], [F2], [F3] and also [A2]). These characterizations turned out to be useful while dealing with a variety of problems. The aim of the first part of the paper is to present a similar characterization (Theorem 2.2) for the absolute multiplicative Borel classes of metric spaces. It seems there has been a lack of such internal characterizations in the literature. For absolute $F_{\sigma \delta}$-spaces, an internal, though not quite simple characterization was given in [Si]. To the best of our knowledge, the only result in this direction, which uses complete sequences of covers - besides Frolík's papers - is the characterization of absolute $F_{\sigma \delta}$-spaces which has been recently given in [JK]. One can regard our internal description of absolute multiplicative Borel classes as a natural extension of the above results due to Frolík and Junnila-Künzi. Complete sequences of covers provide descriptions of "nice" spaces where properties of sets belonging to ( $\sigma$-discrete) covers determine the

Received by the editors December 14, 1995.

1991 Mathematics Subject Classification. Primary 04A15, 54H05, 54C35.

Key words and phrases. Absolute Borel set, function space.

The first author was supported in part by KBN grant 2 P301 02407.

The second author was supported in part by the grant GAČR 201/94/0069 and the grant of the Czech Acad. Sci. 119401. 
properties of the space in a surprisingly simple way (for analytic spaces, there is no restriction on sets; for absolute Borel classes, we refer to theorems stated below). One should recall that complete sequences of covers have found applications in nonseparable descriptive set theory (see e.g. [Ha]).

For a completely regular space $X, C_{p}(X)$ denotes the space of all continuous real valued functions on $X$ with the pointwise convergence topology.

Spaces $X$ and $Y$ are called $l$-equivalent (resp. $u$-equivalent, $t$-equivalent) if $C_{p}(X)$ and $C_{p}(Y)$ are linearly homeomorphic (uniformly homeomorphic, homeomorphic, respectively). So $\sigma$-compactness is known to be preserved by $t$-equivalence (for a more detailed account see Section 4). Arhangel'skiil's problem of whether complete metrizability is preserved by $l$-equivalence was answered affirmatively (for the class of metric spaces) in [BGP]. As recalled above, completely metrizable spaces are precisely the absolute $G_{\delta}$-spaces, but the problem of preservation of other absolute Borel classes by $l$-equivalence was left open in [BGP]. We are going to show that these absolute Borel classes are preserved even by a uniformly continuous surjection $\Phi: C_{p}(X) \rightarrow C_{p}(Y)$ (Theorem 3.4). Let us recall that the map $\Phi: C_{p}(X) \rightarrow C_{p}(Y)$ is uniformly continuous if for every neighborhood $U$ of zero (i.e. constant zero function) in $C_{p}(Y)$ there is a neighborhood $V$ of zero in $C_{p}(X)$ such that, for every $f, g \in C_{p}(X)$ with $(f-g) \in V$ we have $(\Phi(f)-\Phi(g)) \in U$. Our proof technique of Theorem 3.4 is based on some ideas from [Gu2]. This proof does not work for the case of absolute $G_{\delta}$-spaces which we leave as an open problem. However, we obtain preservation results for some other classes of metric spaces (Theorem 3.5).

Let us point out that the class of uniformly continuous maps is essentially larger than the class of linear continuous maps. This may be illustrated by the result of Gul'ko that for all infinite countable compact spaces $X$, the spaces $C_{p}(X)$ are uniformly homeomorphic; this fact does not hold for linear homeomorphisms, see [Gu1].

We will use the following notation for Borel classes.

If $M$ is a metrizable space and $\alpha$ is a countable ordinal then $\mathcal{A}_{\alpha}(M)$ (resp., $\mathcal{M}_{\alpha}(M)$ ) denotes the family of subsets of $M$ that are Borel of additive (resp., multiplicative) class $\alpha$. By $\mathcal{A}_{\alpha}$ (resp., $\mathcal{M}_{\alpha}$ ), $\alpha>1$, we denote the class of spaces that are absolute Borel of additive (resp., multiplicative) class $\alpha$. We refer the reader to $[\mathrm{Ku}]$ and $[\mathrm{Ke}]$ for information concerning Borel classes.

\section{Characterizations of absolute Borel sets}

Definition. Let $X$ be a metrizable space. A sequence $\mathfrak{S}=\left\langle\mathcal{S}_{n}\right\rangle_{n=0}^{\infty}$ of covers of $X$ is said to be complete if the following condition is satisfied: whenever $\mathcal{F}$ is a filter on $X$ such that

$$
\forall n \in \omega: \mathcal{S}_{n} \cap \mathcal{F} \neq \emptyset
$$

then $\mathcal{F}$ has a cluster point in $X$, i.e. $\bigcap\left\{\mathrm{Cl}_{X}(F): F \in \mathcal{F}\right\} \neq \emptyset\left(\mathrm{Cl}_{X}(F)\right.$ is the closure of $F$ in $X$ ).

In the sequel, filters satisfying $\left({ }^{*}\right)$ will be called $\mathfrak{S}$-small. If $\mathcal{S}_{n}$ is the cover by $\frac{1}{n}$-balls, then $\mathfrak{S}$-small filters are precisely Cauchy ones. We will frequently use the following simple observation: If $\left\langle\mathcal{S}_{n}\right\rangle_{n=0}^{\infty}$ is a complete sequence of covers of $X$ and the cover $\mathcal{T}_{n}$ is a refinement of $\mathcal{S}_{n}$, for every $n$, then the sequence $\left\langle\mathcal{T}_{n}\right\rangle_{n=0}^{\infty}$ is also complete. 
The following theorem summarizes known internal characterizations of absolute Borel classes involving complete sequences of covers:

2.0. Theorem (Frolík; Junnila-Künzi).

1. A metrizable space $X$ is an absolute $G_{\delta}$-set iff there is a complete sequence $\left\{\mathcal{S}_{n}\right\}_{n \in \omega}$ of $(\sigma$-discrete) open covers of $X$.

2. A metrizable space $X$ is an absolute $F_{\sigma \delta}$-set iff there is a complete sequence $\left\{\mathcal{S}_{n}\right\}_{n \in \omega}$ of $\sigma$-discrete closed covers of $X$.

In part (1) of the above theorem, one can take $\sigma$-discrete open covers because of the A. H. Stone paracompactness theorem for metrizable spaces, the original Frolík's theorem for topological spaces could not use such covers, of course. A. H. Stone's theorem even enables us to assume without loss of generality that "discreteness" means "uniform discreteness". Recall that a family $\mathcal{D}$ of subsets of a metric space $X$ is said to be uniformly discrete if there is $\delta>0$ such that each $\delta$-ball in $X$ meets at most one element from $\mathcal{D}$. The uniform discreteness is not used in the classical formulation of the Stone theorem (see [En], 4.4.1, 4.4.2). Nevertheless, the standard proof of this theorem (see [En], page $350^{2-4}$ ) shows that, for an arbitrary compatible metric on $X$, every open cover of $X$ has an open $\sigma$-uniformly discrete refinement. This fact is well-known, see e.g. [Is], VII.4.

In fact, we will use Theorem 2.0.(1) as the first step of the inductive proof of Theorem 2.2. In this proof we will also use the following notation and lemma.

Notation. Let $A$ be a subset of a metrizable space $Z$. For every countable ordinal $\alpha>0$ and $W \in \mathcal{A}_{\alpha}(A) \backslash \bigcup\left\{\mathcal{A}_{\beta}(A): \beta<\alpha\right\}\left(W \in \mathcal{A}_{1}(A)\right.$, for $\left.\alpha=1\right)$ we fix $E(W) \in \mathcal{A}_{\alpha}(Z)$ such that $W \subseteq E(W) \subseteq \mathrm{Cl}_{Z}(W)$ and $E(W) \cap A=W$.

2.1. Lemma. Let $A$ be a subset of a metric space $Z$. Let $\alpha>0$ be a countable ordinal and let $\left\{\mathcal{W}_{n}: n \in \omega\right\}$ be a countable family of families of subsets of $A$ such that $\mathcal{W}_{n}$ is uniformly discrete and $\mathcal{W}_{n} \subseteq \mathcal{A}_{\alpha}(A)$, for every $n \in \omega$.

Then there is $P \in \mathcal{M}_{\alpha}(Z)$ such that $A \subseteq P$ and for each finite family $\mathcal{L} \subseteq$ $\bigcup\left\{\mathcal{W}_{n}: n \in \omega\right\}$ we have:

$$
(\bigcap\{E(W): W \in \mathcal{L}\} \cap P \neq \emptyset) \Longleftrightarrow(\bigcap \mathcal{L} \neq \emptyset) .
$$

Proof. As each $\mathcal{W}_{n}$ is uniformly discrete, the family $\left\{E(W): W \in \mathcal{W}_{n}\right\}$ is uniformly discrete as well for each $n \in \omega$.

For finite $J \subseteq \omega$ put

$\mathcal{G}(J)=\left\{\bigcap_{j \in J} E\left(W_{j}\right): W_{j} \in \mathcal{W}_{j}\right.$, for $j \in J$, and $\bigcap_{j \in J} W_{j}=\emptyset$ and $\left.\bigcap_{j \in J} E\left(W_{j}\right) \neq \emptyset\right\}$

and $S(J)=\bigcup \mathcal{G}(J)$.

The family $\mathcal{G}(J)$ is uniformly discrete and $\mathcal{G}(J) \subset \mathcal{A}_{\alpha}(Z)$, hence $S(J) \in \mathcal{A}_{\alpha}(Z)$ and $S(J) \cap A=\emptyset$. So $S=\bigcup\{S(J): J$ is a finite subset of $\omega\} \in \mathcal{A}_{\alpha}(Z)$.

Put $P=Z \backslash S$. We have $P \in \mathcal{M}_{\alpha}(Z)$ and it is easy to verify that $P$ has the other required property.

2.2. Theorem. Let $X$ be a metrizable space and let $\alpha$ be a countable ordinal. For every $\alpha \geq 2$ the following conditions are equivalent (for $\alpha=1$ the conditions (a) and (b) are equivalent):

(a) the space $X$ belongs to the absolute multiplicative class $\mathcal{M}_{\alpha}$, 
(b) there is a complete sequence $\mathfrak{S}=\left\langle\mathcal{S}_{n}\right\rangle_{n=0}^{\infty}$ of $\sigma$-discrete covers of $X$ such that:

$$
\forall(n \in \omega) \exists\left(\alpha_{n}<\alpha\right)\left[\mathcal{S}_{n} \subseteq \mathcal{A}_{\alpha_{n}}(X)\right],
$$

(c) there is a complete sequence $\mathfrak{T}=\left\langle\mathcal{T}_{n}\right\rangle_{n=0}^{\infty}$ of $\sigma$-discrete covers of $X$ such that:

$$
\forall(n \in \omega) \exists\left(\alpha_{n}<\alpha\right)\left[\mathcal{T}_{n} \subseteq \bigcup\left\{\mathcal{M}_{\beta}(X): \beta<\alpha_{n}\right\}\right] .
$$

2.3. Remark. Obviously, for $\alpha=1$, the equivalence (a) $\Leftrightarrow(\mathrm{b})$ in the above theorem is Frolík's theorem 2.0.(1) and the equivalence (a) $\Leftrightarrow(\mathrm{c})$, for $\alpha=2$, is the result of Junnila and Künzi 2.0.(2). Let us point out that in the case of separable spaces $X$ the phrase " $\sigma$-discrete covers" in this characterization means "countable covers". Also, for the nonlimit ordinals $\alpha$ the statements (b) or (c) can be simplified in the natural way, e.g. if $\alpha=\beta+1$, for some $\beta<\omega_{1}$, then we can require in (b) that $\mathcal{S}_{n} \subseteq \mathcal{A}_{\beta}(X)$, for every $n$.

It is clear that, using our characterizations, it is also possible to formulate internal characterizations of absolute additive classes (just use the fact that an absolute Borel set of the additive class $\alpha$ is a countable union of the sets of lower multiplicative classes). As mentioned above, Frolík (see [F4]) proved that a metrizable space $X$ is analytic (i.e. a continuous image of the irrationals) iff there is a complete sequence $\mathfrak{S}=\left\langle\mathcal{S}_{n}\right\rangle_{n=0}^{\infty}$ of countable covers of $X$; notice that there is no restriction on elements of $\mathcal{S}_{n}$.

Proof of Theorem 2.2. (a) $\Rightarrow$ (b) We will prove this implication by induction on $\alpha$. For $\alpha=1$ it follows from Theorem 2.0(1) (in fact, it is enough to take $\sigma$-discrete open covers $\mathcal{S}_{n}$ of $X$ consisting of sets of diameter less than $2^{-n}$ with respect to some compatible complete metric on $X)$.

Suppose $\alpha>1$ and (a) $\Rightarrow$ (b) holds for all $1 \leq \beta<\alpha$. Take $X \in \mathcal{M}_{\alpha}$ and fix a compatible metric $d$ on $X$. Let $Z$ be the completion of $X$. Hence $X \in \mathcal{M}_{\alpha}(Z)$ and $X=\bigcap_{n \in \omega} D_{n}$ where $D_{n} \in \mathcal{A}_{\alpha_{n}}(Z)$ and $1 \leq \alpha_{n} \leq \alpha_{n+1}<\alpha$ for each $n \in \omega$. Each $D_{n}$ is a union of sets $P_{j}^{n}, j \in \omega$, such that $P_{j}^{n} \in \bigcup\left\{\mathcal{M}_{\beta}(Z): \beta<\alpha_{n}\right\}$. Put $S_{j}^{n}=P_{j}^{n} \backslash \bigcup\left\{P_{i}^{n}: i<j\right\}$. Then $D_{n}$ is a disjoint union of sets $S_{j}^{n}$ and we have $S_{j}^{n} \in \mathcal{M}_{\alpha_{n}}(Z) \cap \mathcal{A}_{\alpha_{n}}(Z)$ for every $j \in \omega$.

For each $S_{j}^{n}$ we are going to use the inductive hypothesis for $\alpha_{n}$. This means that we can take a complete sequence $\mathfrak{S}(n, j)=\left\langle\mathcal{R}_{k}^{(n, j)}\right\rangle_{k=n}^{\infty}$ of $\sigma$-discrete covers of $S_{j}^{n}$ such that $\mathcal{R}_{k}^{(n, j)} \subseteq \mathcal{A}_{\alpha_{n}}\left(S_{j}^{n}\right)$ for every $n, j \in \omega$ and each $k \geq n$ (here we only need such a weaker form of (b)). Observe that we also have $\mathcal{R}_{k}^{(n, j)} \subseteq \mathcal{A}_{\alpha_{n}}(Z)$, since $S_{j}^{n} \in \mathcal{A}_{\alpha_{n}}(Z)$. Refining these covers if necessary, we may assume that all covers from $\mathfrak{S}(n, j)$ are $\sigma$-uniformly discrete and that, for every $n, j \in \omega$ and $k \geq n$, the cover $\mathcal{R}_{k}^{(n, j)}$ consists of sets of diameter less than $2^{-k}$.

We define $\mathfrak{S}=\left\langle\mathcal{S}_{p}\right\rangle_{p=0}^{\infty}$ by

$$
\mathcal{S}_{p}=\left\{X \cap \bigcap_{n=0}^{p} R^{n}: R^{n} \in \mathcal{R}_{p}^{\left(n, j_{n}\right)}, \text { for some } j_{n} \in \omega, \text { and }\left(X \cap \bigcap_{n=0}^{p} R^{n} \neq \emptyset\right)\right\} .
$$

Since $\mathcal{R}_{k}^{\left(n, j_{n}\right)} \subseteq \mathcal{A}_{\alpha_{n}}(Z)$, and the sequence $\left(\alpha_{n}\right)$ is nondecreasing, we have $\mathcal{S}_{p} \subseteq$ $\mathcal{A}_{\alpha_{p}}(X)$. The cover $\mathcal{S}_{p}$ is clearly $\sigma$-uniformly discrete for each $p \in \omega$.

It remains to show that $\mathfrak{S}=\left\langle\mathcal{S}_{p}\right\rangle_{p=0}^{\infty}$ is a complete sequence. 
Take an $\mathfrak{S}$-small filter $\mathcal{F}$ on $X$. In particular, $\mathcal{F}$ can have at most one cluster point in $Z$ because diameters of sets in covers $\mathcal{S}_{p}$ converge to 0 as $p \rightarrow \infty$. For each $p \in \omega$, choose $H_{p} \in \mathcal{S}_{p} \cap \mathcal{F}$. We have

$$
H_{p}=X \cap \bigcap_{n=0}^{p} R_{p}^{n} \quad \text { where } R_{p}^{n} \in \mathcal{R}_{p}^{\left(n, j_{n}\right)} \text { for } 0 \leq n \leq p .
$$

Notice that $j_{n}$ does not depend on $p$ as $S_{j}^{n} \cap S_{j^{\prime}}^{n}=\emptyset$ for $j \neq j^{\prime}$. Hence, for each $p \in \omega$ and $0 \leq n \leq p$, we have $H_{p} \subseteq R_{p}^{n} \in \mathcal{R}_{p}^{\left(n, j_{n}\right)}$. This means that $\left\{F \cap S_{j_{n}}^{n}: F \in \mathcal{F}\right\}$ is a base for a $\mathfrak{S}\left(n, j_{n}\right)$-small filter $\mathcal{F}_{n}$ on $S_{j_{n}}^{n}$. As $\lim _{p \rightarrow \infty} \operatorname{diam} R_{p}^{n}=0$, there is a unique cluster point $a_{n}$ of $\mathcal{F}_{n}$ in $S_{j_{n}}^{n} \subset D_{n}$. Clearly, $a_{n}$ is also a cluster point of $\mathcal{F}$ in $Z$. Therefore, for all $n, n^{\prime} \in \omega$, we have $a_{n}=a_{n^{\prime}}=a$ and $a \in \bigcap D_{n}=X$.

(b) $\Rightarrow$ (a) The case of $\alpha=1$ follows from Frolík's theorem (Thm. 2.0(1)). Hence we may assume that $\alpha>1$ and $\alpha_{n}>0$, for all $n$. We fix a compatible metric $d$ on $X$. Let $Z$ be the completion of $X$. For a Borel subset $W$ of $X, E(W)$ has the meaning from the Notation preceding Lemma 2.2.

Suppose $\mathfrak{S}=\left\langle\mathcal{S}_{n}\right\rangle_{n=0}^{\infty}$ is a complete sequence of covers of $X$ as in (b). Again, without loss of generality, we may assume that $\mathcal{S}_{n}$ is $\sigma$-uniformly discrete and $\operatorname{diam}(W)<2^{-n}$ for each $W \in \mathcal{S}_{n}$ and every $n \in \omega$. Let $\mathcal{S}_{n}=\bigcup_{j \in \omega} \mathcal{D}_{j}^{n}$ where $\mathcal{D}_{j}^{n}$ is uniformly discrete for each $j \in \omega$. By our assumptions on $\mathfrak{S}$ we have $\mathcal{D}_{j}^{n} \subseteq \mathcal{A}_{\alpha}(X)$. Therefore we may apply Lemma 2.1 (for $A=X$ and $Z$ as above) to the family $\left\{\mathcal{D}_{j}^{n}: n, j \in \omega\right\}$. Let $P$ be the set satisfying the assertion of 2.1. Since $P \in \mathcal{M}_{\alpha}(Z)$ it is enough to show that $X \in \mathcal{M}_{\alpha}(P)$.

For $W \in \bigcup \mathfrak{S}$, put $F(W)=E(W) \cap P$. Hence $F(W) \in \mathcal{A}_{\alpha_{n}}(P)$ for each $W \in \mathcal{S}_{n}$. For $n, j \in \omega$, put $D_{j}^{n}=\bigcup\left\{F(W): W \in \mathcal{D}_{j}^{n}\right\}$. By the uniform discreteness of $\mathcal{D}_{j}^{n}$ we have $D_{j}^{n} \in \mathcal{A}_{\alpha_{n}}(P)$. Put $D_{n}=\bigcup_{j \in \omega} D_{j}^{n}$. So $D_{n} \in \mathcal{A}_{\alpha_{n}}(P)$ as well.

Let $X^{\prime}=\bigcap_{n \in \omega} D_{n}$. Hence $X^{\prime} \in \mathcal{M}_{\alpha}(P)$ and $X^{\prime} \supseteq X$. Take $x \in X^{\prime}$. For each $n$, there are $i(n)$ and $W_{n} \in \mathcal{D}_{i(n)}^{n}$ such that $x \in F\left(W_{n}\right)$; recall that $F\left(W_{n}\right) \subseteq E\left(W_{n}\right)$. By the property of $P$, the family $\left\{W_{n}\right\}_{n \in \omega}$ is centered. Let $\mathcal{G}$ be the filter on $X$ generated by $\left\{W_{n}\right\}_{n \in \omega}$. By the completeness of $\mathfrak{S}$, the filter $\mathcal{G}$ has a cluster point $p \in X$. As $\operatorname{diam}\left(W_{n}\right)<2^{-n}$, this cluster point is unique and $\operatorname{dist}(p, x)=0$. Hence $x \in X$ and $X^{\prime}=X$.

(b) $\Rightarrow$ (c) Let $\mathfrak{S}$ be the complete sequence of covers as in (b). For every $W \in \mathcal{S}_{n}$, $n \in \omega$, we can represent $W \in \mathcal{A}_{\alpha_{n}}(X)$ as a union of sets $W_{k} \in \bigcup\left\{\mathcal{M}_{\beta}(X): \beta<\right.$ $\left.\alpha_{n}\right\}$, for $k \in \omega$. Then the sequence $\mathfrak{T}=\left\langle\mathcal{T}_{n}\right\rangle_{n=0}^{\infty}$ defined by $\mathcal{T}_{n}=\left\{W_{k}: W \in \mathcal{S}_{n}\right\}$ satisfies (c).

(c) $\Rightarrow$ (b) Trivial.

\section{Borel ClASSES AND UNIFORMLY CONTINUOUS MAPS OF FUNCTION SPACES}

For a space $X$ and a positive integer $n$, by $[X]^{n}$ we denote the space of all subsets of $X$ of the cardinality $n$ equipped with the Vietoris topology. If $X$ is metrizable, then $[X]^{n}$ is also metrizable by the Hausdorff metric.

$C_{p}^{*}(X)$ is the subspace of $C_{p}(X)$ consisting of bounded functions.

3.1. Proposition. Let $X$ and $Y$ be metric spaces and let $\Phi: C_{p}(X) \rightarrow C_{p}(Y)$ (resp. $\Phi: C_{p}^{*}(X) \rightarrow C_{p}^{*}(Y)$ ) be a uniformly continuous surjection. Then $Y$ is a countable union of closed subspaces $Y_{n}, n \in \omega$, such that $Y_{n}$ can be mapped by a perfect map into $[X]^{k_{n}}$, for some $k_{n} \geq 1$. 
Proof. Part of our argument follows closely the reasoning of Gul'ko from the proof of Proposition 1.4 in [Gu2], but for the sake of completeness we include it here.

We will treat jointly the cases of the spaces $C_{p}(X)$ and $C_{p}^{*}(X)$, so we denote by $D(X)$ and $D(Y)$ the domain and the range of the map $\Phi$, respectively. For every $y \in Y$, we define the map $\alpha_{y}: D(X) \rightarrow \mathbb{R}$ by the formula $\alpha_{y}(f)=\Phi(f)(y)$, for $f \in D(X)$. It is obvious that $\alpha_{y}$ is uniformly continuous.

Let $y \in Y$ and $K$ be a finite subset of $X$. We define

$$
\begin{aligned}
& a(y, K)=\sup \left\{\left|\alpha_{y}(f)-\alpha_{y}(g)\right|: f, g \in D(X)\right. \text { such that } \\
& \qquad|f(x)-g(x)|<1 \text { for every } x \in K\} .
\end{aligned}
$$

Obviously, $a(y, \emptyset)=\infty$ since $\Phi$ is onto. By $\mathcal{A}(y)$ we denote the family of all finite subsets $K \subseteq X$ such that $a(y, K)<\infty$. We will prove that $\mathcal{A}(y)$ is nonempty. Using the uniform continuity of $\alpha_{y}$ we find a finite $K \subseteq X$ and an $n \geq 1$ such that $\left|\alpha_{y}(f)-\alpha_{y}(g)\right|<1$ for every $f, g \in D(X)$ with $|f(x)-g(x)|<\frac{1}{n}$ for all $x \in K$. Take $f, g \in D(X)$ such $|f(x)-g(x)|<1$ for all $x \in K$. We put $f_{k}=f+\frac{k}{n}(g-f)$, for $k=0,1, \ldots, n$. Then $f_{0}=f, f_{n}=g$ and $\left|f_{k}(x)-f_{k+1}(x)\right|<\frac{1}{n}$; hence $\left|\alpha_{y}\left(f_{k}\right)-\alpha_{y}\left(f_{k+1}\right)\right|<1$, for $k<n$. Adding up all these inequalities we obtain that $\left|\alpha_{y}(f)-\alpha_{y}(g)\right|<n$ which shows that $K \in \mathcal{A}(y)$.

The family $\mathcal{A}(y)$ has the following property:

(1) $\forall\left(K_{1}, K_{2} \in \mathcal{A}(y)\right)\left[K_{1} \cap K_{2} \in \mathcal{A}(y)\right.$ and $\left.a\left(y, K_{1} \cap K_{2}\right) \leq a\left(y, K_{1}\right)+a\left(y, K_{2}\right)\right]$.

Let us consider arbitrary functions $f, g \in D(X)$ such that $|f(x)-g(x)|<1$ for every $x \in K_{1} \cap K_{2}$. Take $h \in D(X)$ such that $h\left|K_{1}=f\right| K_{1}$ and $h \mid K_{2} \backslash K_{1}=$ $g \mid K_{2} \backslash K_{1}$. Then $|h(x)-g(x)|<1$ for all $x \in K_{2}$; therefore we have $\mid \alpha_{y}(f)-$ $\alpha_{y}(h) \mid \leq a\left(y, K_{1}\right)$ and $\left|\alpha_{y}(h)-\alpha_{y}(g)\right| \leq a\left(y, K_{2}\right)$, and finally $\left|\alpha_{y}(f)-\alpha_{y}(g)\right| \leq$ $a\left(y, K_{1}\right)+a\left(y, K_{2}\right)$.

From (1) it follows that the family $\mathcal{A}(y)$ contains a unique minimal element (with respect to inclusion), we denote it by $K(y)$ and we put $a(y)=a(y, K(y))$.

Let $p$ and $q$ be positive integers. We define

$$
\begin{aligned}
Y(p, q) & =\{y \in Y: \exists(K \in \mathcal{A}(y))[a(y, K) \leq p \text { and }|K| \leq q]\} \text { and } \\
M(p, q) & =\{y \in Y(p, q): \forall(K \subseteq X)[(|K| \leq q-1) \Rightarrow(a(y, K)>2 p)]\} .
\end{aligned}
$$

We also put $M(p)=\bigcup\{M(p, q): q=1,2, \ldots\}$. From the fact that $K(y)$ is minimal we obtain that:

(2) $(p \geq a(y)) \Rightarrow(y \in M(p))$,

(3) $M(p, 1)=Y(p, 1)$ and $M(p, q)=Y(p, q) \backslash Y(2 p, q-1)$, for $q \geq 2$,

(4) $Y=\bigcup\{M(p, q): p, q=1,2, \ldots\}$,

(5) $M\left(p, q_{1}\right) \cap M\left(p, q_{2}\right)=\emptyset$, for $q_{1} \neq q_{2}$.

Let $y \in M(p, q)$. Then there exists a unique $K_{p}(y) \subseteq X$ such that $\left|K_{p}(y)\right|=q$ and $a\left(y, K_{p}(y)\right) \leq p$. This follows from the fact that for all finite $L \subseteq X$ we have $K_{p}(y) \subseteq L$ if $a(y, L) \leq p$. Indeed, if there existed $L$ such that $a(y, L) \leq p$ and $K_{p}(y) \backslash L \neq \emptyset$, then by (1) we would obtain that $a\left(y, K_{p}(y) \cap L\right) \leq 2 p$, contradicting the fact that $\left|K_{p}(y) \cap L\right| \leq q-1$.

The sets $K_{p}(y)$, defined for every $y \in M(p)$, have the following important property:

(6) Let $\left(y_{n}\right)$ be a sequence of points of $Y$ converging to a point $y \in M(p)$ and let $K_{n}$ be finite subsets of $X$ such that $a\left(y_{n}, K_{n}\right) \leq p$, for every $n$. Then for every 
open $U \subseteq X$ such that $U \cap K_{p}(y) \neq \emptyset$ there is an $n_{0}$ such that $U \cap K_{n} \neq \emptyset$ for all $n \geq n_{0}$.

Suppose that $U \cap K_{n}=\emptyset$ for infinitely many $n$. Passing to a subsequence we may assume that $U$ is disjoint from all $K_{n}$. Since $y \in M(p)$ we may find $f, g \in D(X)$ such that $|f(x)-g(x)|<1$, for all $x \in K_{p}(y) \backslash U$, and $\left|\alpha_{y}(f)-\alpha_{y}(g)\right|>2 p$. Take $h \in D(X)$ such that $h|X \backslash U=f| X \backslash U$ and $h\left|K_{p}(y) \cap U=g\right| K_{p}(y) \cap U$. Then $\mid h(x)-$ $g(x) \mid<1$, for all $x \in K_{p}(y)$; hence $\left|\alpha_{y}(h)-\alpha_{y}(g)\right| \leq p$ and $\left|\alpha_{y}(f)-\alpha_{y}(h)\right|>p$. On the other hand $f\left|K_{n}=h\right| K_{n}$, for all $n$, therefore $\left|\alpha_{y_{n}}(f)-\alpha_{y_{n}}(h)\right| \leq a\left(y_{n}, K_{n}\right) \leq p$. Since $\alpha_{y_{n}}(f) \rightarrow \alpha_{y}(f)$ and $\alpha_{y_{n}}(h) \rightarrow \alpha_{y}(h)$, as $n \rightarrow \infty$, passing to the limits we obtain a contradiction with the previous inequality.

From (6) we immediately obtain:

(7) The map $K_{p}: M(p, q) \rightarrow[X]^{q}$ is continuous.

Next, we need the following:

(8) $Y(p, q)$ is closed in $Y$, for all $p, q=1,2, \ldots$.

Let $y_{n} \in Y(p, q)$ and $y \in Y$ be such that $y_{n} \rightarrow y$, as $n \rightarrow \infty$. We take sets $K_{n} \subseteq X$ such that $\left|K_{n}\right| \leq q$ and $a\left(y_{n}, K_{n}\right) \leq p$. Passing to a subsequence we may assume that there exist $K^{0} \subseteq X$ with $\left|K^{0}\right| \leq q$ and a decomposition $K_{n}=K_{n}^{0} \cup K_{n}^{1}$ such that $K_{n}^{0} \rightarrow K^{0}$ (in the Vietoris topology) and every $x \in X$ has a neighborhood $U_{x}$ meeting only finitely many $K_{n}^{1}$ 's. We will show that $a\left(y, K^{0}\right) \leq p$, i.e. $y \in Y(p, q)$.

Consider $f_{0}, g_{0} \in D(X)$ such that $\left|f_{0}(x)-g_{0}(x)\right|<1$ for all $x \in K^{0}$. Take an arbitrary $\varepsilon>0$. Using the uniform continuity of $\alpha_{y}$ we can find $\delta>0$ and a finite $A \subseteq X$ such that $\left|\alpha_{y}(f)-\alpha_{y}(g)\right|<\varepsilon$ for every $f, g \in D(X)$ satisfying $|f(x)-g(x)|<\delta$, for all $x \in A$. We may assume that $\delta<1$ and $K^{0} \subseteq A$. By the property of sets $K_{n}^{1}$ we can find an open $U \subseteq X$ containing $A$ and a number $n_{1}$ such that $U \cap K_{n}^{1}=\emptyset$, for every $n \geq n_{1}$. Let us take $h \in D(X)$ such that $h \mid A \equiv 1$ and $h \mid X \backslash U \equiv 0$. We put $f_{1}=f_{0} h$ and $g_{1}=g_{0} h$. From $f_{1}\left|A=f_{0}\right| A$ and $g_{1}\left|A=g_{0}\right| A$ it follows that $\left|\alpha_{y}\left(f_{1}\right)-\alpha_{y}\left(f_{0}\right)\right|<\varepsilon$ and $\left|\alpha_{y}\left(g_{1}\right)-\alpha_{y}\left(g_{0}\right)\right|<\varepsilon$. We have $\left|f_{1}(x)-g_{1}(x)\right|=\left|f_{0}(x)-g_{0}(x)\right|<1$, for all $x \in K^{0}$. By the continuity of $f_{1}$ and $g_{1}$ there exists an $n_{0}$ such that $\left|f_{1}(x)-g_{1}(x)\right|<1$ for all $x \in K_{n}^{0}$ and $n \geq n_{0}$. We also have $f_{1}(x)=0=g_{1}(x)$ for $x \in K_{n}^{1}$ and $n \geq n_{1}$. Hence $\left|f_{1}(x)-g_{1}(x)\right|<1$ for all $x \in K_{n}$ and $n \geq n_{0}, n_{1}$, therefore $\left|\alpha_{y_{n}}\left(f_{1}\right)-\alpha_{y_{n}}\left(g_{1}\right)\right| \leq p$. Passing to the limit we obtain that $\left|\alpha_{y}\left(f_{1}\right)-\alpha_{y}\left(g_{1}\right)\right| \leq p$. It follows that $\left|\alpha_{y}\left(f_{0}\right)-\alpha_{y}\left(g_{0}\right)\right|<p+2 \varepsilon$. Since $\varepsilon$ is arbitrary we finally conclude that $\left|\alpha_{y}\left(f_{0}\right)-\alpha_{y}\left(g_{0}\right)\right| \leq p$.

From (3) and (8) it follows that $M(p, q)$ is an $F_{\sigma}$-subset of $Y$, for every $p, q \geq 1$. To finish the proof it remains to verify the following:

(9) Let $M \subseteq M(p, q)$ be closed in $Y$, for some $p, q \geq 1$. Then the map $K_{p} \mid M$ : $M \rightarrow[X]^{q}$ is perfect.

For $n \in \omega$, let $y_{n} \in M$ be such that $K_{p}\left(y_{n}\right)$ converges to some $K \in[X]^{q}$. It is enough to show that $\left(y_{n}\right)$ has a convergent subsequence in $Y$ (hence in $M$ ). Suppose the contrary, then $Z=\left\{y_{n}: n \in \omega\right\}$ is closed and discrete in $Y$, therefore every bounded function on $Z$ can be extended to a function from $D(Y)$. We may assume that $y_{n} \neq y_{m}$ for $n \neq m$. Let $\mathcal{A}$ be an uncountable almost disjoint family of infinite subsets of $\omega$, i.e. $A \cap B$ is finite for distinct $A, B \in \mathcal{A}$. For $A \in \mathcal{A}$ let $f_{A} \in D(Y)$ be such that $f_{A}\left(y_{n}\right)=p+1$ if $n \in A$ and $f_{A}\left(y_{n}\right)=0$ for $n \in \omega \backslash A$. We can find $g_{A} \in D(X)$ such that $\Phi\left(g_{A}\right)=f_{A}$. Let $K=\left\{x_{1}, \ldots, x_{q}\right\}$. We put $s_{A}=\left(g_{A}\left(x_{1}\right), \ldots, g_{A}\left(x_{q}\right)\right) \in \mathbb{R}^{q}$. Since $\mathbb{R}^{q}$ is separable we can find distinct $A, B \in \mathcal{A}$ 
such that the distance between $s_{A}$ and $s_{B}$ is less than 1, i.e. $\left|g_{A}\left(x_{i}\right)-g_{B}\left(x_{i}\right)\right|<1$, for $i=1, \ldots, q$. By the continuity of $g_{A}$ and $g_{B}$ there exists an $n_{0}$ such that $\left|g_{A}(x)-g_{B}(x)\right|<1$ for all $x \in K_{p}\left(y_{n}\right)$ and $n \geq n_{0}$. From the definition of the map $K_{p}$ it follows that $\left|f_{A}\left(y_{n}\right)-f_{B}\left(y_{n}\right)\right|=\left|\alpha_{y_{n}}\left(g_{A}\right)-\alpha_{y_{n}}\left(g_{B}\right)\right| \leq p$ for $n \geq n_{0}$. This contradicts the fact that $A \backslash B$ is infinite.

3.2. Corollary. Let $\mathcal{P}$ be a class of (separable) metrizable spaces with the following properties:

(a) $\mathcal{P}$ contains all complete (compact) metrizable spaces,

(b) $\mathcal{P}$ is closed under finite products,

(c) if $M \in \mathcal{P}$ and $N$ is a closed subset of $M$, then $N \in \mathcal{P}$,

(d) if $M$ is a metrizable space which is a countable union of closed subsets $M_{n} \in$ $\mathcal{P}$, then $M \in \mathcal{P}$.

Let $X \in \mathcal{P}$ and $Y$ be a metrizable space. If there exists a uniformly continuous surjection $\Phi: C_{p}(X) \rightarrow C_{p}(Y)\left(\right.$ resp. $\left.\Phi: C_{p}^{*}(X) \rightarrow C_{p}^{*}(Y)\right)$, then $Y \in \mathcal{P}$.

Proof. This corollary can be easily derived from 3.1 using the following two observations.

First, from a result of Klee (see [BP, p. 271], [Kl]) it follows that, for a metric space $X$, the space $X^{n}$ contains an $F_{\sigma}$-subset $A$ such that the map $f: A \rightarrow[X]^{n}$, defined by $f\left(\left(x_{1}, \ldots, x_{n}\right)\right)=\left\{x_{1}, \ldots, x_{n}\right\}$, is a bijection. Let $A=\bigcup_{k=1}^{\infty} A_{k}$, where each $A_{k}$ is closed in $X^{n}$. One can easily verify that the restriction $f \mid A_{k}$ is a closed embedding into $[X]^{n}$. Therefore if $X \in \mathcal{P}$, then also $[X]^{n} \in \mathcal{P}$.

Second, let $Y$ and $Z$ be (separable) metrizable spaces and $f: Y \rightarrow Z$ be a perfect map. Let $\hat{Y}$ be the completion (a metrizable compactification) of $Y$ and let $i: Y \rightarrow \hat{Y}$ be an embedding. Then the diagonal map $f \triangle i$ embeds $Y$ onto a closed subset of $Z \times \hat{Y}$. It follows that $Y \in \mathcal{P}$ if $Z \in \mathcal{P}$.

We need the following standard fact (cf. [DM, Prop. 3.8]):

3.3. Proposition. Let $X$ be a metrizable space such that $X=\bigcup_{n=1}^{\infty} X_{n}, X_{n} \in$ $\mathcal{M}_{\alpha}, \alpha \geq 2$ and $X_{n} \in \mathcal{A}_{\beta}(X)$, where $\beta<\alpha$. Then $X \in \mathcal{M}_{\alpha}$.

Proof. Let $Y$ be a completion of $X$ and let $Y_{n} \in \mathcal{A}_{\beta}$ be such that $Y_{n} \cap X=$ $X_{n}$. Consider $Z=\bigcup_{n=1}^{\infty} Y_{n} \in \mathcal{A}_{\beta} \subseteq \mathcal{M}_{\alpha}$. We have $Y_{n} \backslash X_{n} \in \mathcal{A}_{\alpha}$; hence, $\bigcup_{n=1}^{\infty}\left(Y_{n} \backslash X_{n}\right) \in \mathcal{A}_{\alpha}$ and $X=Z \backslash \bigcup_{n=1}^{\infty}\left(Y_{n} \backslash X_{n}\right) \in \mathcal{M}_{\alpha}$.

3.4. Theorem. Let $X$ and $Y$ be metrizable spaces and let $\Phi: C_{p}(X) \rightarrow C_{p}(Y)$ (resp. $\Phi: C_{p}^{*}(X) \rightarrow C_{p}^{*}(Y)$ ) be a uniformly continuous surjection. If $X \in \mathcal{M}_{\alpha}$ (resp. $X \in \mathcal{A}_{\alpha}$ ), $\alpha>1$, then also $Y \in \mathcal{M}_{\alpha}$ (resp. $Y \in \mathcal{A}_{\alpha}$ ).

Proof. Follows directly from 3.2 and 3.3 .

This theorem answers affirmatively a question from [BGP, p. 882]. Another immediate application of Corollary 3.2 is the following (here $\Sigma_{n}^{1}$ and $\Pi_{n}^{1}$ denote the classes of projective spaces, see [Ke, Sec. 37]):

3.5. Theorem. Let $X$ and $Y$ be separable metrizable spaces and let $\Phi: C_{p}(X) \rightarrow$ $C_{p}(Y)$ (resp. $\Phi: C_{p}^{*}(X) \rightarrow C_{p}^{*}(Y)$ ) be a uniformly continuous surjection. If $X \in \Sigma_{n}^{1}$ (resp. $X \in \Pi_{n}^{1}$ ), $n \geq 1$, then also $Y \in \Sigma_{n}^{1}$ (resp. $Y \in \Pi_{n}^{1}$ ). 


\section{REMARKS}

The results of the paper [BGP] show that the complete metrizability of $X$ is preserved by linear surjections of the function spaces $C_{p}(X)$ and $C_{p}^{*}(X)$. We do not know if this can be generalized to uniformly continuous surjections. Using 3.2 we can show that the class of so-called strongly $\sigma$-complete spaces (i.e. metric spaces which are countable unions of closed completely metrizable subspaces) is preserved by such surjections. Obviously, the class of strongly $\sigma$-complete spaces is strictly larger than the class of completely metrizable spaces.

4.1. Question. Let $X$ and $Y$ be metrizable spaces and let $\Phi: C_{p}(X) \rightarrow C_{p}(Y)$ (resp. $\Phi: C_{p}^{*}(X) \rightarrow C_{p}^{*}(Y)$ ) be a uniformly continuous surjection (resp., a uniform homeomorphism). Is $Y$ completely metrizable if $X$ is so?

Let us recall that for the space of rationals $\mathbb{Q}$ and for the convergent sequence $X=\{0,1,1 / 2,1 / 3, \ldots\}$ the spaces $C_{p}(\mathbb{Q})$ and $C_{p}(X)$ are homeomorphic, see [DGM]. Therefore the complete metrizability is not preserved by homeomorphisms of $C_{p}(X)$. However, we do not know how big the difference in the Borel classes of metrizable spaces $X$ and $Y$ can be if $C_{p}(X)$ and $C_{p}(Y)$ are homeomorphic.

Let us also point out that the $\sigma$-compactness is preserved by continuous surjections $\Phi: C_{p}(X) \rightarrow C_{p}(Y)$ (resp. $\Phi: C_{p}^{*}(X) \rightarrow C_{p}^{*}(Y)$ ), for metrizable $X$ and $Y$. This follows from the fact that for a metrizable space $X, X$ is $\sigma$-compact iff $C_{p}(X)$ (resp. $\left.C_{p}^{*}(X)\right)$ is analytic, see $[\mathrm{Ch}]$ and $[\mathrm{DM}]$. The result of Uspenskiı [Us] implies that, for metrizable spaces, the compactness is preserved by uniformly continuous surjections of function spaces.

In the nonmetrizable case the $\sigma$-compactness (compactness) is preserved by (uniform) homeomorphisms of $C_{p}(X)$, see [O], [Us] or [A1]. But the following simple example shows that these properties are not preserved by continuous linear surjections.

4.2. Example. Let $\omega_{1}$ and $\omega_{1}+1$ be the spaces of ordinals with the usual order topology. Obviously $\omega_{1}+1$ is compact and $\omega_{1}$ is not $\sigma$-compact but the restriction map is a linear surjection of $C_{p}\left(\omega_{1}+1\right)$ onto $C_{p}\left(\omega_{1}\right)$.

If we restrict ourselves to linear surjections, then Corollary 3.2 can be strengthened a little bit and the proof can be essentially simplified. We have the following:

4.3. Proposition. Let $\mathcal{P}$ be a class of metrizable spaces with the following properties:

(a) $\mathcal{P}$ contains the interval $[0,1]$,

(b) $\mathcal{P}$ is closed under finite products,

(c) if $M \in \mathcal{P}$ and $N$ is a closed subset of $M$, then $N \in \mathcal{P}$,

(d) if $M$ is a metrizable space which is a countable union of closed subsets $M_{n} \in$ $\mathcal{P}$, then $M \in \mathcal{P}$.

Let $X \in \mathcal{P}$ and $Y$ be a metrizable space. If there exists a continuous linear surjection $\Phi: C_{p}(X) \rightarrow C_{p}(Y)\left(\right.$ resp. $\left.\Phi: C_{p}^{*}(X) \rightarrow C_{p}^{*}(Y)\right)$, then $Y \in \mathcal{P}$.

Proof. (Sketch) Let $L_{p}(X)$ and $L_{p}(Y)$ denote the dual spaces of $C_{p}(X)$ and $C_{p}(Y)$, respectively. We consider these spaces with the weak* topology, i.e. the topology generated by $C_{p}(X)$ and $C_{p}(Y)$, respectively. It is well-known that $L_{p}(X)$ consists of linear combinations of evaluation functionals, see [A1, Ch. 0.5]. Using the result of Klee mentioned above and results from [A1, Ch. 0.5$]$ it is easy to represent $L_{p}(X)$ 
as a countable union of closed subspaces $Z_{n}, n \in \omega$, such that $Z_{n}$ is homeomorphic to a closed subset of $[0,1]^{k_{n}} \times X^{k_{n}}$, for some $k_{n} \geq 1$ (these homeomorphisms are given by taking appropriate linear combinations of evaluation functionals). Now, we take the canonical embedding $e: Y \rightarrow L_{p}(Y)$ given by evaluation functionals and the dual map $\Phi^{*}: L_{p}(Y) \rightarrow L_{p}(X)$. The composition $\Phi^{*} e$ is an embedding of $Y$ into $L_{p}(X)$. One can easily verify that $\Phi^{*} e \mid\left(\Phi^{*} e\right)^{-1}\left(Z_{n}\right)$ is a closed embedding. The same argument works for the spaces of bounded continuous functions.

This proposition gives us a much easier way to prove the weaker versions of Theorems 3.4 and 3.5 for the linear maps. It also allows us to show that some classes of metrizable spaces $X$ like countable dimensional spaces, strongly countable dimensional spaces, are preserved by linear surjections of function spaces $C_{p}(X)$. Corollary 3.2 and Proposition 4.3 can be compared with earlier general results on preservation of some topological classes under mappings of function spaces, cf. [O, Cor. 2], [A1].

The preservation results from $[\mathrm{BGP}]$ were obtained using the characterization of completeness in terms of complete sequence of covers. Let us point out that using a similar technique it is also possible to apply Theorem 2.2 for the alternative proof of Theorem 3.4 for linear continuous surjections from $C_{p}(X)$ onto $C_{p}(Y)$. Recently Valov [Va] has extended the results of [BGP] to the compact-open topology on $C_{p}(X)$ and also for other function spaces. It is very likely that Theorem 2.2 will allow us to use this technique for analogous extensions for higher absolute Borel classes as well. Surely, it will be treated elsewhere.

4.4. Christensen's theorem on $\mathcal{K}(X)$. For a space $Z, \mathcal{K}(Z)$ denotes the set of compact subsets of $Z$. Let $X$ and $Y$ be metrizable spaces. Christensen [Ch] proved that if $X$ is Polish and there is a map $\Phi: \mathcal{K}(X) \rightarrow \mathcal{K}(Y)$ such that

1. $\Phi(A) \subseteq \Phi(B)$ whenever $A \subseteq B, \quad A, B \in \mathcal{K}(X)$,

2. if $C \in \mathcal{K}(Y)$, then there is $A \in \mathcal{K}(X)$ such that $C \subseteq \Phi(A)$,

then $Y$ is Polish, as well. Recall that $\mathcal{K}(\mathbb{Q})$ equipped with the Hausdorff metric is not analytic, see $[\mathrm{Ke}$, Ex.27.4]. However, we can define the map $\Phi: \mathcal{K}(\mathbb{Q}) \rightarrow$ $\mathcal{K}(\mathcal{K}(\mathbb{Q}))$, satisfying the above conditions $(1)$ and $(2)$, by the formula: $\Phi(A)=$ $\{L \in \mathcal{K}(\mathbb{Q}): L \subseteq A\}$, for $A \in \mathcal{K}(\mathbb{Q})$. This example shows that Christensen's result cannot be extended to the class of $\sigma$-compact spaces and higher Borel classes. Christensen's theorem and an extension of it to the nonseparable case obtained in $[\mathrm{BGP}]$ were used therein for an alternative proof that complete metrizability is preserved by linear continuous surjections from $C_{p}(X)$ to $C_{p}(Y)$. Due to the above example this approach cannot be extended to prove such preservation results for higher Borel classes. So the uniform (or linear) structure of $C_{p}(X)$ is a finer tool for an investigation of $X$ than the lattice $\mathcal{K}(X)$. For other references and properties encoded by $\mathcal{K}(X)$ we refer to $[\mathrm{Fr}]$.

4.5. Remarks on Wijsman topologies. We will conclude with some remarks concerning the descriptive complexity of Wijsman hyperspaces which are related to the function spaces $C_{p}(X)$. For a metric space $(X, \rho)$, the Wijsman hyperspace topology $w_{\rho}$ on the set $2^{X}$ of all nonempty closed subsets of $X$ is defined by identifying each $F \in 2^{X}$ with a function $\phi_{F}(x)=\operatorname{dist}(x, F)$, for each $x \in X$, and considering $\left\{\phi_{F}: F \in 2^{X}\right\}$ as a subspace of $C_{p}(X)$ (see [Be]). Recall that the space $\left(2^{X}, w_{\rho}\right)$ is metrizable (and then separable) iff the space $(X, \rho)$ is separable. Therefore, we restrict ourselves to separable metric spaces in this remark. 
The topology $w_{\rho}$ does depend on the given metric but the identity map id : $\left(2^{X}, w_{\rho}\right) \rightarrow\left(2^{X}, w_{\sigma}\right)$ is Borel measurable for equivalent metrics $\rho$ and $\sigma$. In fact, the preimage of any open set is $F_{\sigma}$, i.e. the identity map is a Borel isomorphism of the first class.

As observed in $[\mathrm{Ba}],\left(2^{X}, w_{\rho}\right)$ is analytic whenever $(X, \rho)$ is. On the other hand, $C_{p}(X)$ is analytic iff $X$ is $\sigma$-compact [Ch]. For absolutely Borel hyperspaces we have a similar situation: It follows immediately from nontrivial results in [SR] that $\left(2^{X}, w_{\rho}\right)$ is Borel iff $(X, \rho)$ is the union of a Polish space and a $\sigma$-compact set. However, the situation is nice when $X$ is of a low Borel class: It is proved in [Co] that $\left(2^{X}, w_{\rho}\right) \in \mathcal{M}_{1}$ provided $(X, \rho) \in \mathcal{M}_{1}$. As observed in [CLP], a necessary condition for $\sigma$-compactness of $\left(2^{X}, w_{\rho}\right)$ is the finiteness of the set of points where $(X, \rho)$ is not locally compact. In particular, $\left(2^{\mathbb{Q}}, w_{\rho}\right)$ is not $\sigma$-compact for any compatible metric $\rho$ on the space of rationals $\mathbb{Q}$. One can easily show that $\left(2^{X}, w_{\rho}\right) \in \mathcal{M}_{2}$ provided $X$ is $\sigma$-compact. Following the argument from [SR, proof of Theorem 1] it can also be proved that $\left(2^{X}, w_{\rho}\right) \in \mathcal{M}_{2}$ if $X$ is the union of a Polish space and a $\sigma$-compact set.

\section{ACKNOWLEDGMENT}

The authors are grateful to Henno Brandsma, Tadeusz Dobrowolski, Jan van Mill and Roman Pol for valuable suggestions and comments.

\section{REFERENCES}

[A1] A. V. Arkhangel'skiū, Topological Function Spaces, Kluwer Academic Publishers, Dordrecht, 1992. MR 92i:54022

[A2] - On topological spaces which are complete in the sense of Čech, Vest. Mosk. Univ. (1961), 37-40. MR 24:A1110

[BGP] J. Baars, J. de Groot, J. Pelant, Function spaces of completely metrizable spaces, Trans. Amer. Math. Soc. 340 (1993), 871-879. MR 94f:54036

[Ba] A. Barbati, The hyperspace of an analytic metrizable space is analytic, Proc. 11th Int. Conf. on Topology (Trieste 1993); Rend. Ist.Mat. Trieste 25 (1993), 15-21. MR 96c:54047

[Be] G. Beer, Topologies on closed and closed convex sets, Kluwer Academic Publishers, Dordrecht, 1993. MR 95k:49001

[BP] C. Bessaga and A. Pełczyński, Selected Topics in Infinite-Dimensional Topology, PWN, Warszawa, 1975. MR 57:17657

[Ch] J.P.R. Christensen, Topology and Borel Structure, North-Holland Publishing Company, Amsterdam, London, 1974. MR 50:1221

[Co] C. Costantini, Every Wijsman topology relative to a Polish space is Polish, Proc. Amer. Math. Soc 123 (1995), 2569-2574. MR 95j:54012

[CLP] C. Costantini, S. Levi, J. Pelant, On compactness in hyperspaces, in preparation.

[DGM] T. Dobrowolski, S. P. Gulko and J. Mogilski, Function spaces homeomorphic to the countable product of $\ell_{f}^{2}$, Topology Appl. 34 (1990), 153-160. MR 91c:57024

[DM] T. Dobrowolski and W. Marciszewski, Classification of function spaces with the pointwise topology determined by a countable dense set, Fund. Math. 148 (1995), 35-62. MR 96k:54017

[En] R. Engelking, General topology, PWN, Warszawa, 1977. MR 58:18316b

[Fr] D. H. Fremlin, Families of compact sets and Tukey's ordering, Atti Sem. Mat. Fis. Univ. Modena 39 (1991), 29-50. MR 92c:54032

[F1] Z. Frolik, Generalization of the $G_{\delta}$-property of complete metric spaces, Czech. Math. J. 10 (1960), 359-379. MR 22:7100

[F2] - Topologically complete spaces, Comment. Math. Univ. Carol. 1 (1960), 1-3.

[F3] A contribution to descriptive theory of sets and spaces, General Topology and its Relations to Modern Analysis and Algebra, J. Novák, ed., Academia, Prague, 1962, pp. 157-173. MR 26:3002 
[F4] A survey of separable descriptive theory of sets and spaces, Czech. Math. J. 20 (1970), 406-467. MR 42:1660

[Gu1] S. P. Gul'ko, The space $C_{p}(X)$ for countable infinite compact $X$ is uniformly homeomorphic to $c_{0}$, Bull. Pol. Acad. Sci. 36 (1988), 391-396. MR 92e:54011

[Gu2] — O ravnomernykh gomeomorfizmakh prostranstv nepreryvnykh funktsiu, Baku International Topological Conference (1987), Trudy Mat. Inst. Steklova 193 (1990) (Russian); English transl. in Steklov Inst. Math. (1993), 87-93. MR 95f:54017

[Ha] R. W. Hansell, Descriptive topology, Recent progress in general topology, M. Hušek and J. van Mill, editors, North-Holland, Amsterdam, 1992, pp. 275-315. CMP 93:15

[Is] J. R. Isbell, Uniform spaces, Math. Surv. 12, A.M.S., Providence, Rhode Island, 1964. MR 30:561

[JK] H. J. K. Junnila and H. P. A. Künzi, Characterizations of absolute $F_{\sigma \delta}$-sets, preprint.

[Ke] A. S. Kechris, Classical Descriptive Set Theory, Springer-Verlag, New York, 1995. MR 96e:03057

[Kl] V. L. Klee, On the Borelian and projective types of linear subspaces, Math. Scand. 6 (1958), 189-199. MR 21:3752

$[\mathrm{Ku}] \quad$ K. Kuratowski, Topology I, Academic Press and PWN, New York and London, 1966. MR 36: 840

[O] O. G. Okunev, O slabou topologii soprazhennogo prostranstva i otnoshenii t-ekvivalentnosti, Mat. Zametki 46 (1989), 53-59 (Russian); English transl., Weak topology of a dual space and a t-equivalence relation, Math. Notes 46 (1989), 534-536. MR 91h:46008

[SR] J. Saint Raymond, La structure borélienne d'Effros est-elle standard?, Fund. Math. 100 (1978), 201-210. MR 80g:54044

[Si] W. Sierpiński, Sur une définition topologique des ensembles $F_{\sigma \delta}$, Fund. Math. 6 (1924), $24-29$.

[Us] V. V. Uspenskiı̌, A characterization of compactness in terms of uniform structure in a function space, Uspekhi Matem. Nauk 37 (1982), 183-184. MR 83k:54025

[Va] V. Valov, Linear mappings between function spaces, preprint.

Vrije Universiteit, Faculty of Mathematics and Computer Science, De Boelelaan 1081 A, 1081 HV Amsterdam, The Netherlands

Current address: Institute of Mathematics, University of Warsaw, Banacha 2, 02-097 Warszawa, Poland

E-mail address: wmarcisz@cs.vu.nl

Mathematical Institute of the Czech Academy of Sciences, Žitná 25, 11567 Praha 1, Czech Republic

E-mail address: pelant@mbox.cesnet.cz 